HazMat; mass casualties; prevention

Blood Purification Therapy for Crush Syndrome: An Analysis of 50 Cases Caused by the Great Hanshin Earthquake

Sbigeru Shiono, MD; Shinzo Mukainaka, MD; Tatsuro Kai, MD; Muneo Obta, MD

Osaka Prefectural Senri Critical Care Medical Center, Suita-City, Osaka Japan

Introduction: The Great Hanshin Earthquake resulted in more than 300 patients with crush syndrome. Severe cases were managed using blood purification therapy including hemodialysis (HD), continuous hemodiafiltration (CHDF), and plasma exchange (PE). This study was performed to analyze the use of blood purification therapy on patients with the crush syndrome at trauma centers outside of the disaster area.

Methods: A retrospective review was conducted at seven trauma centers in Osaka.

Results: A total of 50 crush syndrome patients were transferred and treated at these centers. Out of these 50 patients, three patients died: two patients from shock in the early stage, and one from septic multi-organ failure (MOF) in the late stage. Of the 50 patients, 38 patients (76\%) developed acute renal failure (defined as serum creatinine $\geq 2 \mathrm{mg} / \mathrm{dl}$ ); 12 patients (24\%) had non-oliguric renal failure, and 26 other patients $(52 \%)$ had oliguric renal failure. Blood purification was performed in 36 patients $(72 \%) ; 17$ patients (34\%) were managed using only HD; 13 patients (26\%) were managed using CHDF in the acute phase and with HD in the late phase; one patient was managed by only with CHDF until death; and five patients (10\%) were managed using both $\mathrm{PE}$ and $\mathrm{HD}$.

The relationship between peak value of serum creatine kinase $(\mathrm{CK})$, which indicated the mass of injured muscle, for the first three days and development of acute renal failure was examined.

1) no renal failure

n

(1)

10

peak value of CK (IU/I) (mean \pm SD)

2) non-oliguric renal failure
a) no need for HD
b) need for $\mathrm{HD}$
3
8
$53,123 \pm 30,801$
$86,969 \pm 48,423$
$144,671 \pm 61,460$
5
uric renal failure

There is a significant difference between peak value of $\mathrm{CK}$ of these four groups. The duration of renal failure and peak value of $\mathrm{CK}$ also has a significant correlation. Conclusion: The blood purification therapy on the crush syndrome patients after the Great Hanshin Earthquake was reviewed. The severity of the renal failure correlated significantly with peak value of $\mathrm{CK}$.

Key Words: acute renal failure; blood purification therapy; continuous hemodiafiltration; serum creatinine; crush syndrome; hemodialysis; multi-organ failure; plasma exchange

\section{Ketamine in Anesthesia Emergency and Disaster Medicine Experiences during the UN Mission in Somalia}

\section{Dr. Peter Enders}

German Federal Armed Forces Central Hospital, Department of Anesthesia and Intensive Care Medicine, Koblenz, Germany

Since 1969, ketamine has played an important role in emergency medicine. Depending on the dose administered, it can be used either for analgesia or anaesthesia. The known relative contraindications may be neglected under these conditions. Ketamine gives the unique possibility to achieve sufficient analgesia when administered by intramuscular injection. Corresponding to numerous publications in emergency medicine, ketamine has stood the test when used for patients in shock. Its utility in these patients is related to its good analgesia with great therapeutic width and the lack of depression of the heart-circulation system and respiration.

In the literature, it is known that in missions of the German Red Cross in hospitals in Thailand, Lebanon, Pakistani and India from 1979 through 1984, more than the half of the patients requiring anaesthesia were anaesthetized using Ketamine. Similarly, its use was reported from the Yom-Kippur War in 1973. Experiences in Turkey during the earthquake mission of German Federal Armed Forces have given ketamine an outstanding role in missions conducted under difficult circumstances. Because of free capacity, the German MASH in Somalia provided humanitarian assistance to the Somalian population. From July to December 1993, 540 operations were conducted using anesthesia of which 91\% employed ketamine-midazolam anesthesia with spontaneous breathing. The decision for use of this type of anesthesia, which in Germany is mostly reserved to Emergency and Disaster Medicine, was established in the realization of the need to integrate a modern medical system in the given socio-economic conditions of a developing country. Examples of these experiences will be reported.

Key Words: analgesia; anesthesia; disaster medicine; emergency medicine; ketamine

\section{Session 7B: Miscellaneous \\ Chairpersons: G.R. Leon (USA) \\ M. Lahad (Israel)}

\section{Information Support System for Medical Aid to Burned Victims in Disasters}

A.N. Putintsev; L.I. Gerasimova; V.N. Zhizbin

Central Institute of Traumatology and Orthopaedics, Sklifosovsky Emergency Aid Institute, Moscow, Russia

Our experience in liquidation of major disasters and medical consequences showed serious difficulties in organization of medical aid to victims. Medical aid required the elaboration of an Information Support System (ISS) for 
medical decision-making in case of disproportions between the large number of victims and the restricted capacities of the medical service. The ISS is a multimedia, hypertext, computer program which is intended to retrieve the necessary information to provide accurate timely diagnosis and to choose optimal treatment for burn patients, especially in mass flame disasters.

Using this system, a physician can retrieve data needed for determination of burn shock severity and shock prognosis on the basis of clinical, functional, and laboratory data as well as the details of optimal anti-shock therapy. The ISS also allows retrieval of required information on the methods of respiratory function, restoration in thermo-chemical injuries of the respiratory system, methods of parenteral and enteral probe feeding, modern tactics of skin plasty, necessary information for triage, and medical aid to victims at the scene, as well as at medical evacuation staging, particularly for specialized hospitals. It includes hundreds of color videoimages of victims with burns of different severities and localization, a glossary of more than 700 terms, and audio support and digital videoclips that illustrate different treatment methods in acute and subsequent stages of burn illness.

The search for necessary information can be accomplished by means of "keywords." In-context-stipulated search with logical conditions also is available. The system can be used not only as an electronic guide for selection of medical actions in cases of disaster, but also can be implemented as a very good visual aid for training. More detailed information on the ISS application can be obtained from the Central Institute of Traumatology and Orthopaedics (Facsimile: 095-154-3139).

Key Words: burns; decision; disaster; information support; multimedia training

\section{An Introduction to the Global Health Disaster Network (GHDNet)}

G. Ocbi; Y. Sbirakawa; K. Nitta; Y. Watob; E. Pretto; $A$. Sekikawa; R.E. La Porte

Department of Emergency Medicine, Ehime University School of Medicine, Ehime, Japan and the Safar Center for Resuscitation Research, University of Pittsburgh, Pittsburgh, Pennsylvania USA

In May 1995, the Global Health Disaster Network (GHDNet) was started with the concept of a disasteroriented project of the Global Health Network (GHNet) at the University of Pittsburgh. The goal of the GHDNet is networking people who are involved in disaster management. Those with various backgrounds, i.e., health-care professionals in disaster and emergency medicine, paramedics and firefighters, public health specialists, are welcome to the network. Being started primarily in Japan, the project will be extended to the United States and the rest of the world. The GHDNet project in Japan consists of following three components: 1) Establishment of $W W W$ bome pages-In July 1995, the GHDNet Home Page (http://hypnos.m.ehime-u. ac.jp/GHDNet) was launched as the first Japanese home page focused on disaster and emergency medicine.
Moreover, we have helped to start more than 20 home pages for disaster related organizations and individuals. The WADEM home page (http://hypnos.m.ehime-u. ac.jp/GHDNet/WADEM) is one of the home pages on our server;

2) Networking people by mailing lists-We started four mailing lists: The Mailing List for Disaster and Emergency Medicine; The Mailing List for Fire and Disaster Prevention; The Mailing List for Japan Red Cross; and The Disaster and Public Health Mailing List. We also are active members of the World NGO Network (WNN) and Inter C-Net which are mailing lists for NGO activities and for public officers in charge of disaster management; and

3) Networking people through medical associations-We maintain home pages for the following medical associations: The World Association for Disaster and Emergency Medicine (WADEM); The Japanese Association for Acute Medicine; and The Japanese Association for the Surgery of Trauma.

It will be our mission to make a link from WWW home pages for these associations and mailing lists to the classical systems for urgent information transmission, i.e., telephone and facsimile.

Key Words: disaster medicine; Internet; telecommunication; www

\section{Quality Management System Implementation within the Viennese Red Cross Ambulance and Disaster Service}

Christoph Redelsteiner, BSW, EMT-P

Wien, Germany

The Vienna Red Cross (VRC) offers various social and medical services to the Viennese community. To assure quality of care, the VRC has implemented a Quality Management System and accomplished successful ISO 9001 certification in October 1996.

An arm of the VRC is its Ambulance Service that provides ALS and BLS Emergency Medical Services, convalescent services, transport of handicapped children and family practitioner-response services. About 130,000 responses are provided per year. Some 600 VRC providers of different levels (drivers, emergency medical technicians, physicians) and from different labor backgrounds (employed, civil servants and volunteers) team up to serve the public.

To further improve quality in such a diverse organizational structure, special "quality teams" have been established. The team consists of EMS providers, managers, and members from other involved VRC departments, e.g., administration, public relation, billing. The teams also developed a quality assurance system according to the 20 ISO 9001 standards as a basis for further Total Quality Management efforts.

So far, staff members have been trained by internal quality system auditors, internal quality audits have been conducted, and all staff members have received information, education, and training in quality management issues. Specialty trained "Quality Assurance Partners" 